\title{
Two-Photon Spectroscopy on Rubidium Vapor Contained in Porous Glass
}

\section{Espectroscopía de dos fotones en vapor de Rubidio contenido en Vidrio Poroso}

\author{
L. Amy ${ }^{1 *}$ L. Lenci ${ }^{1}$, S. Villalba ${ }^{1}$, H. Failache ${ }^{1}$, A. Lezama $^{1}$ \\ 1. Instituto de Física, Facultad de Ingeniería, Universidad de la República, J. Herrera y Reissig 565, \\ 11300 Montevideo, Uruguay \\ (*) E-mail: lamy@fing.edu.uy
}

Received: 02/12/2016 Accepted: 23/11/2017

DOI: $10.7149 /$ OPA.50.4.49061

\begin{abstract}
:
We studied spectroscopicaly the fluorescence of a long-lived state of Rubidium for the atomic vapor confined to the micrometric interstices of porous glass cells. The system is studied with the aim of investigating the spectroscopic consequences of the confinement of atoms. The exited level is reached through atom-photon transition. Radiation fields interacting with the atoms in the pores of glass are diffuse due to the propagation in this highly scattering medium. The fluorescence spectra obtained have unusual lineshapes that are well described with a simple theoretical model, considering the diffuse characteristic of the light field. The fluorescence spectra lineshape measured do not exhibit effects due to the three dimensional confinement, however, this effect is noticeable when the fluorescence decay time curves are observed. They show a reduction in its decay time compared with the signal in a cell without confinement.
\end{abstract}

Key words: Porous Glass; Two-Photon Excitation; Spatial three-dimensional Confinement; Diffuse light

\section{RESUMEN:}

En el presente trabajo se estudia la respuesta en fluorescencia de un vapor de Rubidio confinado en los intersticios de una celda de vidrio poroso, sistema altamente difusivo para la luz. El interés es el de estudiar las consecuencias espectroscópicas asociadas al confinamiento espacial de los átomos. La transición seleccionada posee un tiempo de vida medio del estado excitado mayor al tiempo medio de vuelo de los átomos en el poro. Este estado atómico es alcanzado mediante la excitación del átomo por dos campos de diferentes frecuencias. Los espectros de fluorescencia obtenidos presentan formas inusuales, y son bien descriptos por un modelo teórico sencillo que contempla el carácter difuso de la luz. Si bien los mencionados espectros no dan información del confinamiento 3D de los átomos, la acción del confinamiento se ve claramente reflejada al medir el tiempo de decaimiento de la señal de fluorescencia, en donde se observa un acortamiento del tiempo con respecto a la señal de átomos sin confinar.

Palabras clave: Vidrio Poroso; Excitación a dos fotones; Confinamiento espacial en 3D; Campo difuso

\section{REFERENCES AND LINKS}

[1] L. Amy, L. Lenci, S. Villalba, H. Failache, A. Lezama, "Two-Photon excitation of rubidium atoms inside porous glass", Phys. Rev. A 96, 043819 (2017)

[2] S. Briaudeau, D. Bloch, and M. Ducloy, "Sub-Doppler spectroscopy in a thin film of resonant vapor", Phys. Rev. A59, 3723 (1999)

[3] S. Villalba, H. Failache, L. Lenci, "Non linear atomic spectroscopy inside a random porous medium", Phys. Rev. A 90, 052518 (2014) 
[4] D. S. Wiersma, M. P. van Albada, and A. Legendijk, "Coherent Backscattering of Light from Amplifying Random Media" Phys. Rev. Lett. 75, 1739, (1995)

[5] Q. Bauduin, N. Mercadier, V. Guarrera, W. Guerin, and R. Kaiser, "A cold-atom random laser", Nat. Phys, 9, 357 (2013)

[6] S. Villalba, A. Laliotis, L. Lenci, D. Bloch, A. Lezama, and H. Failache, "Sub-Doppler Resonance in the backscattered light from random porous media infused with Rb vapor", Phys. Rev. A 89, 023422 (2014)

[7] Paul R. Berman and Vladimir S. Malinovsky, Principles of laser spectroscopy and Quantum Optics, Princeton, Princeton University Press, (2011)

\section{Introduction}

Atoms confined to small volumes have spectroscopic responses different from those found in standard cells. Atoms in thin cells consisting of two parallel windows separated by several microns have been widely studied. It enables to obtain sub-Doppler spectra when linear interaction between atoms and light is carried out [1]. When the separation between windows is about the optical wavelength, enhancement of the coherent transient atomic response, due to Dicke narrowing is observed [2]. The inclusion of atomic systems in miniaturized photonic devices also needs a better understanding of effects of confinement that would allow a proper management of their side effects.

At the same time that spectroscopy of atoms under confinement grew in interest, the use of diffuse light in spectroscopy was also studied. Disorder and irregularity in optical media are also essential properties of some optical and spectroscopic systems under study like random laser [3,4].

In this work, we studied alkali atomic vapor confined into micrometric pores in porous glass. Due to the highly scattering characteristic of the porous medium, atoms deep inside the media interact with a diffuse optical field.

We also study the consequences of the 3D confinement on the fluorescence signal, we choose an atomic excited level which has a mean lifetime that exceeds the mean flight-time of the atom inside the pore. We study the fluorescence line-shapes, and relate they singular line-shapes with the diffuse characteristic of the optical field interacting with the atoms.

\section{Experimental Setup}

Our study is based on Rubidium confined in a porous glass cell. The detailed construction of the cells was presented in [5]. The porous medium was made by sintered glass powder inside a glass tube. It was classified into different grain size by meshes and decantation columns, that would define the interstices pore size (typically from $10 \mu \mathrm{m}$ to $100 \mu \mathrm{m}$ ). Porous glass cell containing Rubidium vapor are shown at Fig.1. It consists on a cylindrical $6 \mathrm{~mm}$ diameter glass tube containing ground glass at its end. The system was sintered to create a rigid porous block, where the rubidium would penetrate. A Rubidium dot was distilled inside the cell before it was sealed under vacuum. During the measure, the cell was heated in order to increase the atomic vapor density in the pores.

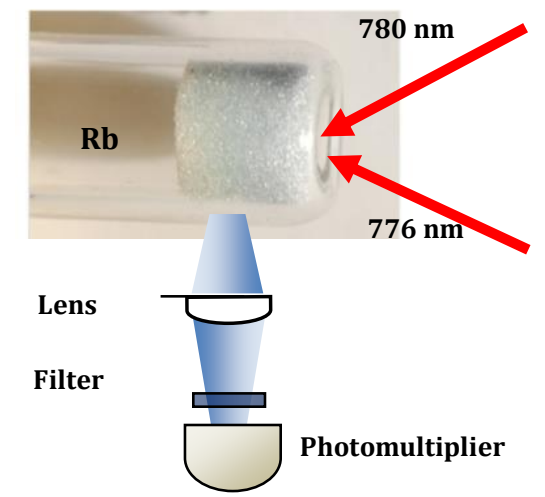

Fig.1. Experimental setup showing a porous glass Rb cell. The lens collects part of the fluorescence, making an image on the detector of a portion of the side surface of the cell. The filter has an narrow transmission centered in the wavelength of interest $(420 \mathrm{~nm})$. 
We considered an atomic state $\left(5 D_{3 / 2}\right)$ whose mean lifetime $(240 \mathrm{~ns})$ is larger than the mean flight time of the atom in the pores. The mean-flight time of atoms contained in $10 \mu \mathrm{m}$ pores cells is about $50 \mathrm{~ns}$. Moreover, we detected the fluorescence signal produce at $420 \mathrm{~nm}$ during the decay of the $6 P_{3 / 2,1 / 2}$ which is populated after de-excitation of $5 D_{3 / 2}$ and whose lifetime is around 120ns. The mechanism of populating the $6 P_{3 / 2,1 / 2}$ level is shown at Fig.2. First, the $5 S_{1 / 2}$ was pumped by a $780 \mathrm{~nm}$ diode laser to the $5 P_{3 / 2}$ level. Then, a second laser couples the $5 P_{3 / 2}$ with the $5 D_{3 / 2}$. That level decay, through the $6 P_{1 / 2}(5 \mu m$ emission, completely absorbed by the glass), to the ground level, giving the fluorescence signal of interest.

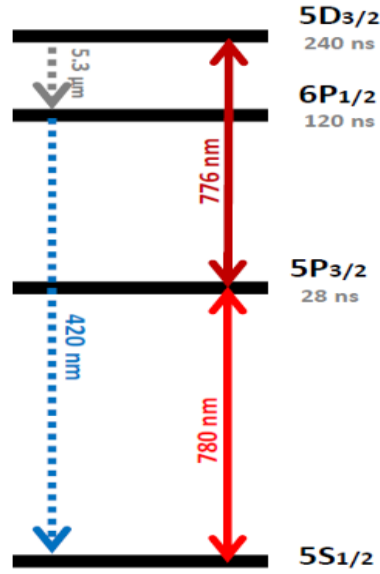

Fig. 2. Atomic levels, coupling fields, and mean lifetimes concerned with the experiment

Both light sources were extended cavity diode lasers. A saturation absorption setup was used as a frequency reference for the pumping laser, and the coupling one was controlled by the scaning system of the laser source, previously calibrated. Laser beams go into the cell by its flat side, as shown in Fig.1. In order to assure the randomness of the light field, only some part of the fluorescence that is far enough from the incident point (aboutn $3 \mathrm{~mm}$ ) was collected through its cylindrical side surface. The signal coming out from the cell was filtered with a $420 \mathrm{~nm}$ filter, and the fluorescence photons were detected by a photomultiplier tube. The incident beams had a power of $15 \mathrm{~mW}$ and $10 \mathrm{~mW}$ for the probe and coupling respectively, but its power was significantly reduced after some micrometers propagating inside the cell.

\section{Model}

As a result of the diffusive effect that the medium has on the light field, atoms that are in pores deep inside the sample interact with a field who has random wavevector, polarization and phase. Moreover, as both fields follow inside the medium different paths and are scattered idependently, they have a relative phase that varies randomly inside the pores. Base on this random fluctuation of the relative phase, coherent effects could be neglected (atomic coherence and two-photon coherent contribution) and the exited state population was considered proportional to the step-wise two photon contribution [6].

On our model, the pump wavector, $k_{p}$, is chosen to be directed along the $y$ axis of a cartesian basis, and the coupling wavector $\left(k_{c}\right)$ direction forming an angle $\theta$ with respecto to $k_{p}$ and located on the $x y$ plane.

With this coordinate system, the velocity distribution of atoms is:

$$
N\left(v_{x}, v_{y}\right)=\frac{N_{o}}{2 \pi \sigma^{2}} e^{-\left(\frac{v_{x}^{2}+v_{y}^{2}}{2 \sigma^{2}}\right)}
$$

Where $N_{o}$ is the number of total atoms and $\sigma^{2}=k_{B} T / m, k_{B}$ the Boltzmann constant, $T$ the temperature, $m$ the atom mass.

We would now consider that the fluorescence amplitude is proportional to the step-wise two photon contribution calculates by the product of two single photon transition Lorentzian resonances corresponding to the two atomic transitions. We introduce the Doppler effect for the two different propagation direction for the light fields $k_{p}$ and $k_{c}$, and we weight the contribution of each atomic velocity by the distribution in Ec. 1. The fluoresce amplitude in terms of fields detuning is then,

$$
A\left(\delta_{p}, \delta_{c}\right) \propto \frac{N_{o}}{2 \pi \sigma^{2}} \iint_{-\infty}^{\infty} e^{-\left(\frac{v_{x}^{2}+v_{y}^{2}}{2 \sigma^{2}}\right)} \frac{1}{\gamma_{p}^{2}+\left(\delta_{p}+k_{p} v_{y}\right)^{2}} \frac{1}{\gamma_{c}^{2}+\left(k_{c} v_{x} \sin \theta+\delta_{c}-\delta_{p} \cos \theta\right)} d v_{x} d v_{y}
$$


Where $\gamma_{p}$ and $\gamma_{c}$ are the width of the pumping and coupling dipole transition.

The expression of (2) can be simplified if the optical transitions widths $\gamma_{p}$ and $\gamma_{c}$ are neglected in comparison to the Doppler width. If $\gamma_{p, c} / k \ll \sigma$, the Lorentzians can be replaced by Delta functions, and (2) can be replaced by

$$
A\left(\delta_{p}, \delta_{c}\right) \propto \frac{N_{o}}{2 \pi D^{2}} \int_{0}^{\pi} e^{-\left(\frac{\left(\delta_{p} \sin \theta\right)^{2}+\left(\delta_{c}-\delta_{p} \cos \theta\right)^{2}}{D^{2} \sin \theta^{2}}\right)} d \theta ;
$$

Where the simultaneous resonance condition for the two fields $\left(k_{o} v_{y}=\delta_{p} ; k_{1} v_{x} \sin \theta=\delta_{c}-\delta_{p} \cos \theta\right)$ was imposed. Neglecting small differences between $k_{p}$ and $k_{c}$ we set $k_{p} \cong k_{c}=k_{o}$. We also introduce $D=$ $\sqrt{2} k \sigma$, the Doppler half width. To take into account the diffuse characteristic of the light fields, the expression obtained on the limits imposed before can be generalized considering the isotropy of the space of the $k_{p}$ vector, and changing base to a polar one. With the introduction of the two polar angles, $\theta$ and $\phi$, and integrating on that variables, assuming a uniform probability distribution of the probe waves over a solid angle, we obtain:

$$
A\left(\delta_{p}, \delta_{c}\right) \propto \frac{N_{o}}{2 \pi D^{2}} \operatorname{erfc}\left(\max \left(\left|\delta_{p}\right|,\left|\delta_{c}\right|\right)\right) ;
$$

Figure 3 shows some spectra calculate using this model. The pumping field frequency is scanned, for different detuning of the coupling field. The shapes of the spectrums are the consequence of the diffuse characteristic of the light. As would be seen on the next section, the spectra predicted by this model have the same plateau like lineshapes that are found in the measurements when large detunings of the probe beam are considered.
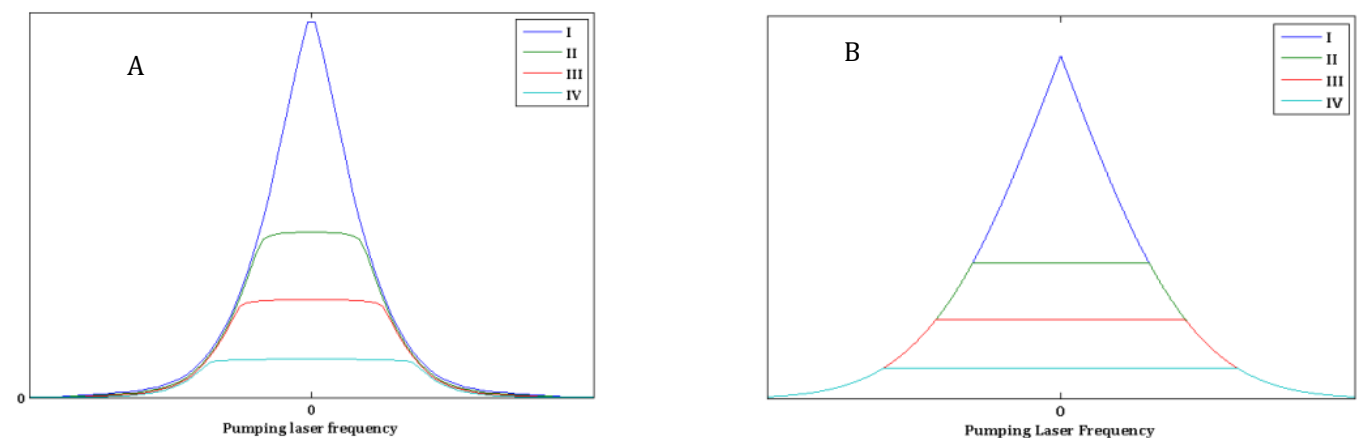

Fig. 3. Spectra lineshapes calculated with A) The model containing the Lorentzians and B) The model under the aproximation of the delta functions. In both curves, the detuning for the coupling 776 laser are: I)0, II)0.6, III)0.85, IV)1.2, referred to the Doppler width

It is interesting to observe that the same analytical model was considered to describe another different physical process. The frequency redistribution of radiation function in dense atomic media through the radiation trapping can be also described by Ec. 3 and Ec. 4 .

\section{Measurements}

\section{4.a. Fluorescence Spectra}

The spectra for $100 \mu \mathrm{m}$ and $10 \mu \mathrm{m}$ pore cells, scanning the780 $\mathrm{nm}$ pump laser, and for different coupling detuning frequencies are shown in Fig. 4. They exhibit a Gaussian shape for detunings near resonance, changing to a plateau lineshape when detuning increase. All the measurements are in accordance with the model presented previously. As it was mentioned before, the flat lineshapes are the consequence of the diffuse characteristic of the light fields.

Comparing Fig 4.a and Fig 4.b corresponding to the measurements for pore sizes of $100 \mu \mathrm{m}$ and $10 \mu \mathrm{m}$ respectively, the spectra do not have any essential difference that could be attributed to an effect of confinement. Measurements of the decay time were made to evidence the atomic spatial confinement on the cells. Those are presented on the next subsection. 

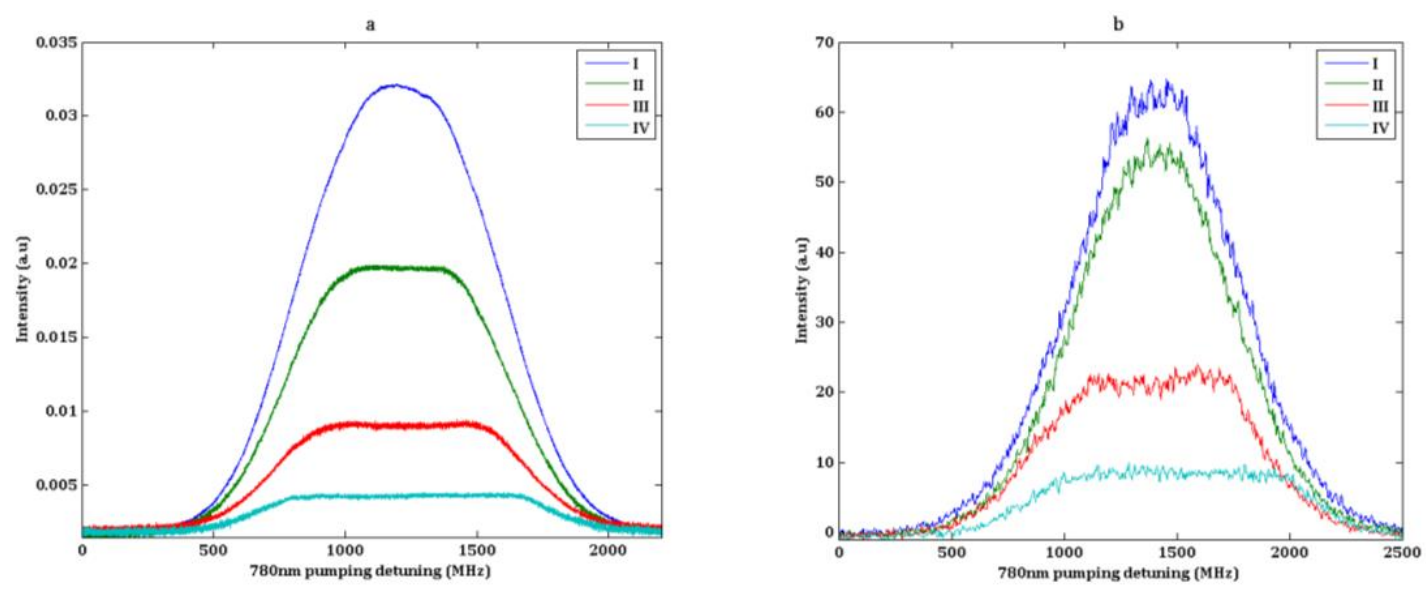

Fig. 4: Fluorescence spectra measured scanning the pumping field for a) $100 \mu \mathrm{m}$ pores cells and b) $10 \mu \mathrm{m}$ pores cells, for different coupling detunig frequencies (a.I: $0 \mathrm{MHz}$, a.II: $214 \mathrm{MHz}$, a.III: $352 \mathrm{MHz}$, a.IV: $464 \mathrm{MHz}$ and b.I: $0 \mathrm{MHz}$, b.II:246MHz, b.III: $477 \mathrm{MHz}$, b.IV: $632 \mathrm{MHz})$.

\section{4.a. Fluorescence Decay Time}

The decay time of the level $5 \mathrm{D}_{3 / 2}$ (through the $6 \mathrm{P}_{3 / 2,1 / 2}$ ) was measured to evidence some effect of the spatial confinement. We compare the decay time inside the porous cell, with pore dimensions of about $50 \mu \mathrm{m}$, with the decay time of the atoms on the same cells but unconfined. The coupling laser was switched off, and the blue fluorescence signal was detected. The transient evolution of the fluorescence intensity is shown on Fig. 4.

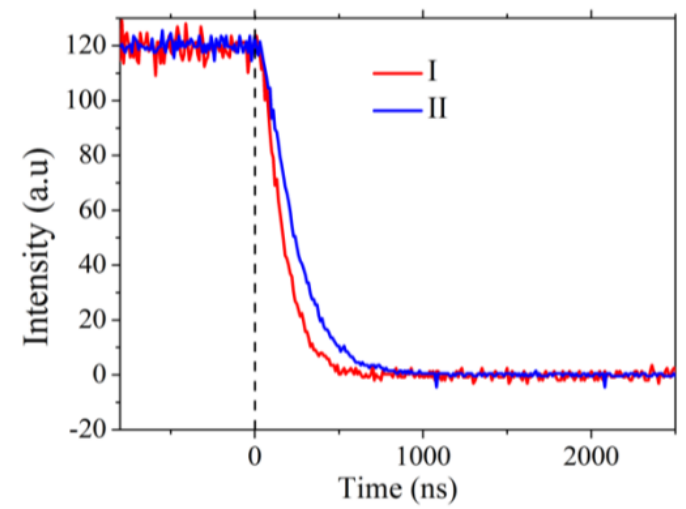

Fig. 5: Decay time of the fluorescence signal for I) Confined (120ns) and II) unconfined atoms (180ns), when the coupling laser is sweetched off.

The decay time was measured fitting the asymptotic decay of fluorescence. However, for the confined atoms, the decay time was $\tau_{c}=120 \mathrm{~ns}$, this significant reduction is explained by the confinement of atomic motion. A decay time of $\tau_{u}=180 n s$ was obtained when fitting the curve for unconfined atoms. Observe that the time measured for the unconfined vapor is shorter than the mean lifetime of the levels $5 D_{3 / 2}$ and $6 P_{3 / 2,1 / 2}$. We explain that difference by the presence of some impurities in the cell.

\section{Conclusions}

We make two-photon spectroscopic measurements of the fluorescence signal of Rubidium atoms confined in porous glass cells. We observe unusual fluorescence line-shapes, that were well explained by a simple model. The model takes into account the diffuse characteristic of both light fields, and coincides mathematically with the Redistribution Frequency Function, a different physical phenomena never measured experimentally. We also measured the effect of the 3D confinement on the decay time of the atoms. All the studies made, give us a better understanding of this original medium. Applications such as random lasers and Rydberg atoms inside the pores will be explored.

\section{Acknowledgements}

We acknowledge financial support from ECOS-Sud, CSIC, PEDECIBA, CAP of Universidad de la República and RIAO/OPTILAS. 